\title{
Anatomically plausible illusory posture affects mental rotation of body parts
}

\author{
Silvio Ionta - Anna Sforza - Mariko Funato • Olaf Blanke
}

Published online: 20 September 2012

(C) Psychonomic Society, Inc. 2012

\begin{abstract}
During mental rotation (MR) of body parts, people internally simulate the movement of their corresponding body segments. These sensory-motor mechanisms render MR sensitive to proprioceptive information (e.g., posture). Similar mechanisms can alter illusory hand ownership following synchronous visuotactile stimulation (e.g., the rubber hand illusion [RHI]). In the present study, we first showed that illusory ownership for a fake hand can also be induced when the posture of the fake hand (palm-up) does not correspond with the subject's physical hand posture (palm-down). Then we tested whether illusory ownership for a fake hand in such a posture impacts the MR of hands carried out immediately and repeatedly after the RHI. The results showed that MR was altered for the view corresponding to the fake hand's posture, but not for other views. Additionally, these effects depended on illusory ownership, as only synchronous visuotactile stimulation was found to lead to these changes, characterized by a modulation of the rotation-dependent profile of MR response times. These findings show that similar sensory-motor mechanisms are recruited during the MR of hands and illusory hand ownership manipulated through multisensory mismatch, and that bottom-up visuotactile stimulation interferes with highlevel imagery processes.
\end{abstract}

\footnotetext{
S. Ionta $(\bowtie) \cdot$ A. Sforza $\cdot$ M. Funato $\cdot$ O. Blanke

Laboratory of Cognitive Neuroscience, Brain-Mind Institute,

École Polytechnique Fédérale de Lausanne (EPFL),

Station 15 ,

1015 Lausanne, Switzerland

e-mail: silvio.ionta@epfl.ch

S. Ionta $\cdot$ O. Blanke

Center for Neuroprosthetics, School of Life Sciences, EPFL,

Lausanne, Switzerland

O. Blanke

Department of Neurology, University Hospital,

Geneva, Switzerland
}

Keywords Embodied cognition · Cognitive control

Mental imagery is a cognitive task that determines the activation of central representations and can cause an almost-perceptive experience, even in the absence of appropriate sensory-motor information (Munzert \& Zentgraf, 2009). In healthy subjects, mental imagery and the execution of a movement share similar temporal and kinematic properties (Decety, Jeannerod, \& Prablanc, 1989; Sirigu et al., 1996). The standard way to objectively measure mental imagery is provided by mental rotation (MR) of visually presented stimuli. MR can be performed with threedimensional objects (Shepard \& Metzler, 1971), alphanumeric characters (Corballis \& Sergent, 1989), human bodies (Parsons, 1987a), and human body parts (Parsons, 1987b), while response times (RTs) and accuracy are recorded. In classic MR paradigms concerning body parts, subjects are asked to judge the laterality of body parts (e.g., hands) presented in different orientations with respect to the visual vertical (Sekiyama, 1982).

Stimulus orientation plays a central role in the MR of hands, leading to progressively increasing RTs for stimuli presented from $0^{\circ}$ to $180^{\circ}$ (clockwise), and decreasing RTs for stimuli presented from $180^{\circ}$ to $0^{\circ}$ (Parsons, 1994; Parsons et al., 1995). The stimulus view also affects MR of hands, leading to longer RTs for stimuli presented in uncommon views (Petit, Pegna, Mayer, \& Hauert, 2003; Thayer, Johnson, Corballis, \& Hamm, 2001). In addition to the main effects of stimulus orientation and view, these two factors also interact in modulating MR (Shenton, Schwoebel, \& Coslett, 2004; ter Horst, van Lier, \& Steenbergen, 2010). This combined effect leads to the so-called awkwardness effect-in which the orientation-dependent distribution of the RT profile is affected by the familiarity of the stimulus views (Corradi-Dell'Acqua \& Tessari, 2010). In addition, according to the so-called 
medial-over-lateral advantage (MOLA; Funk \& Brugger, 2008), MR is faster for rotations toward the midsagittal plane (medial rotations) and longer for rotations away from that plane (lateral rotations; Coslett, Medina, Kliot, \& Burkey, 2010; Funk \& Brugger, 2008; Gawryszewski, Silvados-Santos, Santos-Silva, Lameira, \& Pereira, 2007; Parsons, 1994; ter Horst, Jongsma, Janssen, van Lier, \& Steenbergen, 2012). These findings suggest that subjects internally simulate the movement of their own body part in order to match the position of the stimulus (Parsons, 1994; Sirigu \& Duhamel, 2001 ) by adopting motor imagery in first-person perspective (for a review, see Corradi-Dell'Acqua \& Tessari, 2010) and thus rendering the imagery sensitive to anatomical constraints (Fourkas, Ionta, \& Aglioti, 2006). Compatible with the reliance on such sensory-motor mechanisms, MR of hands is sensitive to proprioceptive information (e.g., body posture), leading to highly specific modulations of RTs as a function of the subject's hand posture during MR of hands (Ionta \& Blanke, 2009; Ionta, Fourkas, Fiorio, \& Aglioti, 2007). This body of evidence supports the idea that central body representations are influenced by peripheral factors such as posture, and further suggests that bodily representation and imagery are based on sensory-motor and multisensory mechanisms (Funk \& Brugger, 2008).

The integration of multisensory input (including the proprioceptive information conveyed by posture) also plays an important role in maintaining the balance between the individual and the environment (Damasio, 2000; Gallagher, 2005; Jeannerod, 2006), is important for the body schema (Berlucchi \& Aglioti, 1997), and is linked to some aspects of self-consciousness, such as body ownership (Blanke \& Metzinger, 2009; Haggard, Taylor-Clarke, \& Kennett, 2003; Jeannerod, 2007). For example, the "rubber hand illusion" (RHI; Botvinick \& Cohen, 1998) is an extensively used experimental protocol for manipulating hand ownership or self-attribution of a fake hand via multisensory conflicts. In this illusion, after observing a rubber hand being stroked synchronously with one's own (hidden) hand, subjects report illusory self-attribution of the rubber hand (Aimola Davies, White, Thew, Aimola, \& Davies, 2010; Armel \& Ramachandran, 2003; Pavani, Spence, \& Driver, 2000; Tsakiris \& Haggard, 2005). According to a recent multisensory model of the RHI, the sufficient conditions for the illusion to occur are (1) that the rubber hand be positioned in an anatomically plausible position and (2) that synchronous visuotactile stimulation be applied within the peripersonal space (for a review, see Makin, Holmes, \& Ehrsson, 2008). Notably, these conditions apply even when the postures of the subject's hand and the rubber hand are incongruent (Makin, Holmes, \& Zohary, 2007). The classic effects of the RHI are synchrony-related changes in subjectively reported self-attribution of the rubber hand (measured with questionnaires), and/or changes in implicitly measured illusory recalibrations of the experienced position of one's own hand toward the rubber hand ("proprioceptive drift"). These effects suggest that following the RHI, an illusory positional recalibration of the subject's hand takes place, so that the hand is "felt" as being closer to the rubber hand.

In addition, neuroimaging studies have suggested that MR of body parts and RHI recruit similar brain networks. In particular, motor imagery and execution activate partially overlapping regions (Grèzes \& Decety, 2001; Munzert, Lorey, \& Zentgraf, 2009), including the premotor cortex (Gerardin et al., 2000; Ionta, Ferretti, Merla, Tartaro, \& Romani, 2010) and the parietal cortex (Overney \& Blanke, 2009; Porro et al., 1996). Similar to the brain activity related to the MR of body parts, during the RHI premotor and inferior parietal cortex are again activated (Ehrsson, Holmes, \& Passingham, 2005; Ehrsson, Spence, \& Passingham, 2004). In particular, the premotor cortex plays a central role in bodily illusions (Lenggenhager, Halje, \& Blanke, 2011; Naito, Ehrsson, Geyer, Zilles, \& Roland, 1999), the inferior parietal cortex is activated during the induction phase of the RHI (Ehrsson et al., 2004), and the RHI is reduced following transcranial magnetic stimulation over the inferior parietal cortex (Kammers et al., 2009).

However, although both MR and RHI are based on sensory-motor and multisensory mechanisms and engage partially overlapping brain networks, their reciprocal influence has not been studied. Adding such information could help to extend current models of sensory-motor and multisensory integration, as well as to advance the existing literature on bodily illusions. In the present study, we attempted for the first time to combine the RHI and MR setups, in order to test the effects of illusory postural changes on an implicit index of bodily processing, the MR of body parts. We hypothesized that, besides the common brain activation, if MR of body parts is sensitive to postural changes and if the RHI induces illusory postural recalibrations, the RHI should affect the MR of body parts. In order to test this hypothesis, we investigated the influence of illusory hand ownership (induced via visuotactile stimulation) on the MR of hands in a within-subjects design. We expected that the MR of hands would be modulated by illusory hand ownership as a function of the synchrony between visuotactile stimulation of the hidden (physical) and seen (fake) hands. In order to test for this effect, we placed the (visible) fake hand in a different but anatomically plausible posture (palmup view), as compared to the stroked (hidden) hand of the subject, which was placed in a palm-down position. We stroked synchronously and asynchronously the fake and hidden hands and tested the MR of the hands immediately after illusion induction. We expected that, similarly to the effect of real postural changes on the MR of hands shown from congruent views (de Lange, Helmich, \& Toni, 2006; 
Ionta \& Blanke, 2009), illusory ownership of the fake hand in palm-view would lead to synchrony-related MR facilitation for hands presented from the palm-view, and not for hands presented from another, control view (dorsum-view). We further expected that the rotation-dependent MOLA-like profile of the MR of hands as a function of the stimulus view would be further modulated by the RHI.

\section{Experiment 1}

\section{Method}

Subjects A group of 42 healthy subjects (16 female, 26 male) 19-26 years of age ( $M=23.8$ years, $S D=2.9$ years) were enrolled in the experiment. All of the subjects were righthanded according to a standard handedness inventory (Briggs $\&$ Nebes, 1975). The experimental protocol was approved by the local ethics committee and written informed consent was obtained prior to participation. The experiment was carried out in accordance with the ethical standards of the 1964 Declaration of Helsinki.

Rubber hand illusion Subjects were seated in front of a device specifically designed to induce the RHI (Lopez, Lenggenhager, \& Blanke, 2010). The device was placed on a table in front of the subjects, at the midchest level, and consisted of a wooden frame with a two-way mirror on the top plane and a flat surface as the bottom plane. Subjects put their hands on the bottom plane, under the mirror. In order to hide the subjects' hands, the left and right thirds of the mirror were obscured by placing two black sheets under the mirror. In the middle of the bottom plane was placed a realistic right fake hand, in a palm-up posture and aligned to the subjects' midsagittal plane. On the wall in front of the fake hand device was a 52-in. flat TV screen, positioned vertically about $1 \mathrm{~m}$ from the subject's eyes (Fig. 1a).

During all blocks, the hands of the subjects were positioned inside the wooden frame of the rubber hand device, on the right and left sides (Fig. 1b). Both subject's hands were in palm-down posture (opposite the rubber hand posture; Fig. 1b). The right hand was positioned on a 5$\mathrm{cm}$-high support that elevated the hand and that left the palm free for stimulation (Fig. 1c). The rubber hand was visible by the subjects only when the lights under the mirror (bottom lights) were on and the lights above the mirror (top lights) were off (Fig. 1d). When the bottom lights were off and the top lights were on, the two-way mirror reflected the roof (Fig. 1e). This setup enabled us to let the subjects see the rubber hand during the visuotactile stimulation (bottom lights on, top lights off) and to hide the rubber hand during the subsequent MR task (bottom lights off, top lights on).
During the visuotactile stimulation, subjects fixated a rubber hand being stroked by the experimenter on the palm ("visual stroking"). According to the experimental design, the palm of the subject's hand was stroked by the experimenter ("tactile stroking") either synchronously or asynchronously with respect to the visual stroking. In order to avoid any potential confound due to the contribution of visuotactile stimulation per se on performance in the imagery task, in accordance with standard procedure (Sforza, Bufalari, Haggard, \& Aglioti, 2010), we used a control condition (hereafter the "baseline" condition) in which the subject's hand was not stroked and only the rubber hand was visible and stroked by the experimenter. Note that both the rubber hand and the hidden stroked hand were right hands and that both were stroked on the palm.

In order to measure self-attribution of the rubber hand, after the synchronous and asynchronous conditions, subjects completed a five-item questionnaire after the MR task that was adapted from the original RHI questionnaire (Botvinick \& Cohen, 1998). Items $1-3$ were related to the illusory selfattribution of the fake hand (target-Q items), and Items 4 and 5 were administered for control (control-Q items; Table 1). Subjects indicated their level of agreement for each of the five items by placing a mark along a 7-point Likert scale ranging from 1 (I totally disagree) to 7 (I totally agree), with respect to both the synchronous and the asynchronous stroking conditions (in counterbalanced order).

Mental rotation The experimental stimuli (Fig. 2) consisted of naturalistic pictures of the hands, presented one at a time on the vertical TV screen, that had been selected from previous studies (e.g., Ionta, Perruchoud, Draganski, \& Blanke, 2012). The left hands were mirror images of the right ones. The stimuli could be presented in one of two different views (dorsum-view or palm-view) and oriented in one of four clockwise orientations from upright $\left(0^{\circ}, 90^{\circ}\right.$, $180^{\circ}$, or $270^{\circ}$ ). The upright orientation was defined as fingers pointing upward $\left(0^{\circ}\right)$. All of the stimuli covered a visual angle of about $17^{\circ}$.

Immediately after the visuotactile stimulation $(2 \mathrm{~min})$, visual stimuli were presented on the TV screen (Fig. 1a). Stimulus presentation and response recording were controlled by the E-Prime2 software (Psychology Software Tools Inc., Pittsburgh, PA). At the beginning of each trial a fixation cross was presented for $1 \mathrm{~s}$. After the fixation cross, one hand picture was presented. The subjects were asked to verbally judge, as quickly and accurately as possible, the laterality (left or right) of the presented hand. The hand remained visible on the screen until a verbal response was given. RTs were automatically recorded by a microphone connected to the computer and positioned in front of the subjects. Accuracy was manually recorded by the experimenter. Subjects' gaze was monitored online. The order of 
Fig. 1 Experimental setup. a Subjects sat in front of the rubber hand illusion device with a TV screen positioned in front of them. A two-way mirror was positioned on the top plane of the device, and a microphone on the bottom plane recorded the subject's verbal response times. b Subject's hands were in a palmdown posture. The rubber hand (right arrow in panel b) was positioned close to the subject's right hand (left arrow in panel b), but in a palm-up posture. Both the rubber hand and the subject's right hand were stroked by the experimenter. c The subject's right hand was positioned on a 5cm-high support, in order to give the experimenter access to the palm to provide tactile stimulation. The red arrow in panel c indicates the sites of tactile stimulation. $\mathbf{d}$ The rubber hand was visible to subjects only when the bottom lights were on and the top lights were off. e When the bottom lights were off and the top lights were on, the rubber hand was not visible, and the two-way mirror reflected the white roof. The green lines indicate the same device's corner during both illumination conditions (darkness, d; light, e)
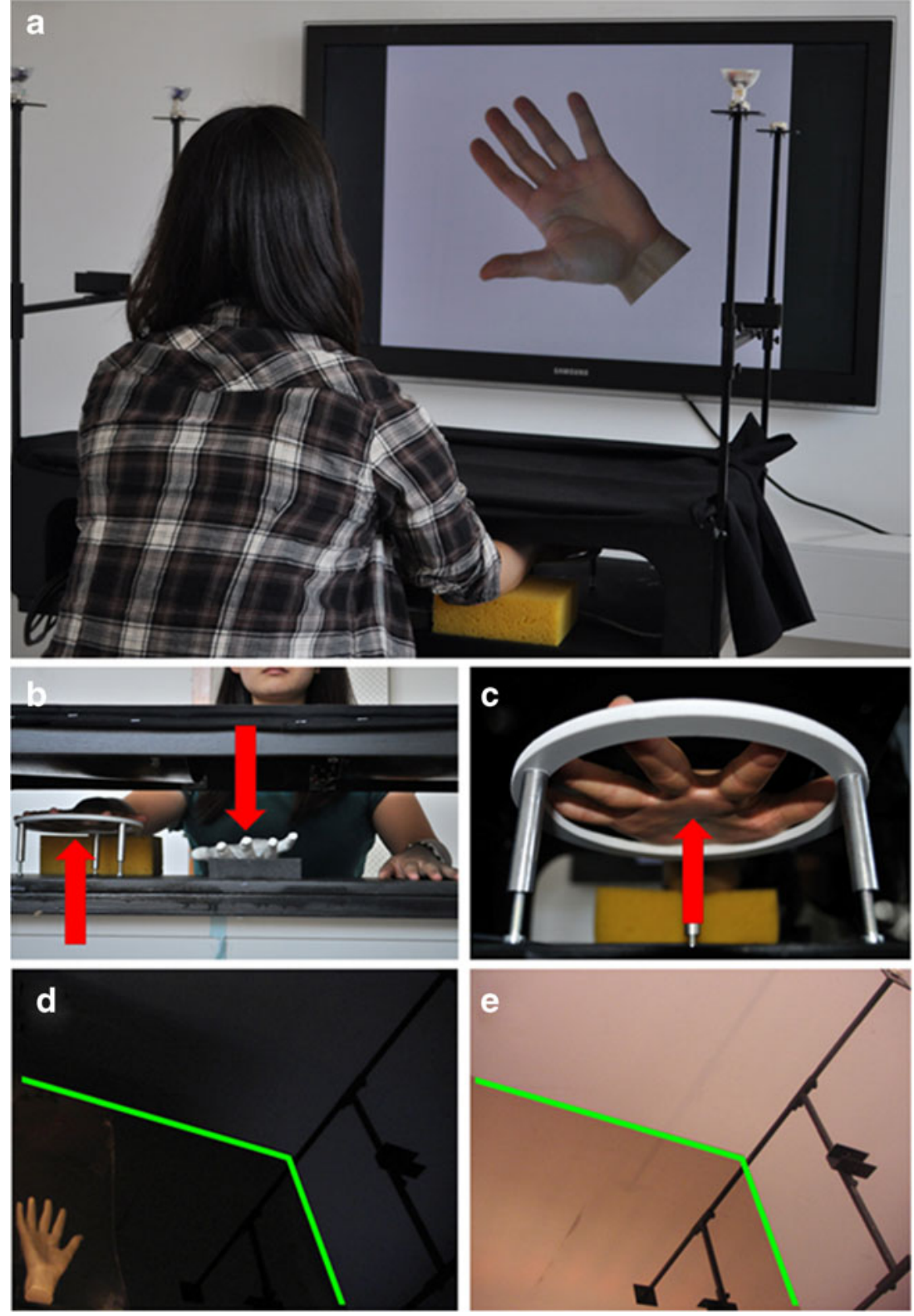

the conditions and stimuli was counterbalanced across subjects. Six blocks of stimuli were presented within the

Table 1 Subjective reports - Statements included in the questionnaire

Statements

Q1. It seemed like I was feeling the touch in the location where I saw the rubber hand being touched.

Q2. It seemed like the touch I felt was caused by the finger touching the rubber hand.

Q3. I felt as if the rubber hand were my hand.

Q4. It felt as if my (real) hand were turning "rubbery."

Q5. The rubber hand began to resemble my own (real) hand, in terms of shape, skin tone, or some other visual feature. experimental session, two blocks for each condition, according to the type of visuotactile stimulation (synchronous, asynchronous, or baseline). Each block contained 16 pictures of hands, including each laterality (eight left- and eight right-lateralized stimuli), two views (eight dorsum-view and eight palm-view stimuli), and four orientations $\left(0^{\circ}, 90^{\circ}, 180^{\circ}\right.$, and $270^{\circ}$, with four stimuli each). Within a block, the same orientation was presented no more than twice in sequence. The effect of the RHI quickly fades over time, therefore we used relatively short blocks. In order to compensate for the consequent small number of repetitions for each stimulus within each subject, we used a large sample of subjects $(N=42)$.

Statistics As an index of the RHI, the scores obtained for target-Q and control-Q in both the synchronous and 

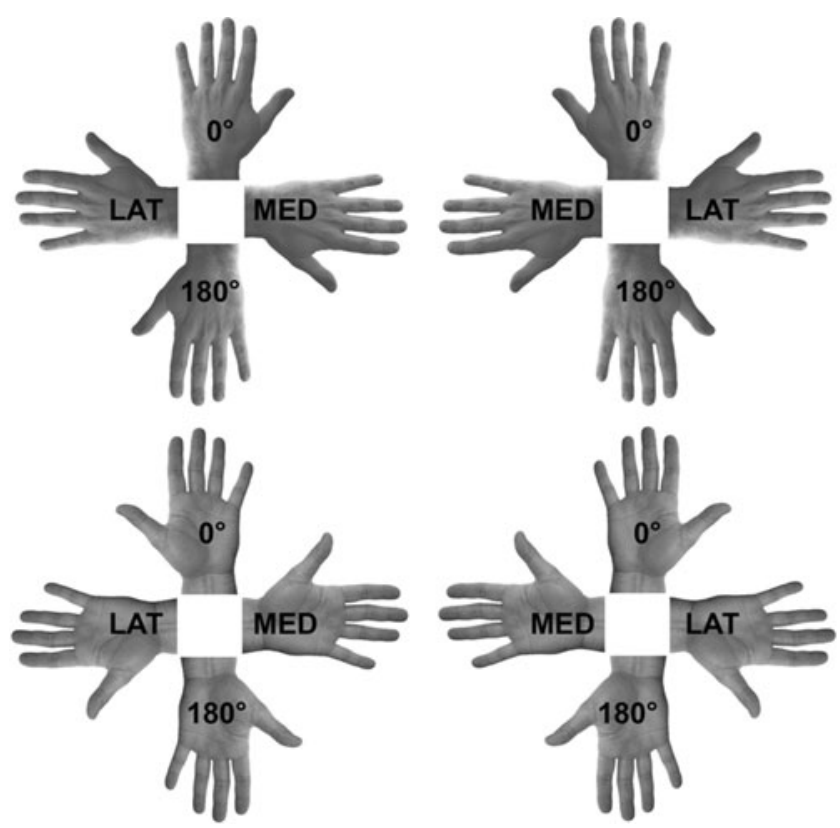

Fig. 2 Mental rotation stimuli. Naturalistic pictures of hands were presented visually. The left hands were mirror images of the right ones. Hands were depicted in one of two different views (dorsum-view or palmview) and oriented in one of four rotations. The upright orientation $\left(0^{\circ}\right)$ was defined as the fingers pointing upward. Lateral rotations (LAT) included right hands at $90^{\circ}$ and left hands at $270^{\circ}$. Medial rotations (MED) comprised right hands at $270^{\circ}$ and left hands at $90^{\circ}$. Upsidedown rotations depicted hands with the fingers pointing downward $\left(180^{\circ}\right)$

asynchronous stroking conditions were merged and compared using paired $t$ tests with Bonferroni correction (Palluel, Aspell, \& Blanke, 2011).

Previous studies had shown that in the MR of hands, RTs are particularly affected by the stimulus view and orientation (Bonda, Petrides, Frey, \& Evans, 1995; Cooper \& Shepard, 1975; de Lange et al., 2006; Parsons, 1987b, 1994; Sekiyama, 1982). Therefore, we analyzed only RTs, which were defined as the time between the onset of the stimulus and the subject's verbal response. We excluded from the analysis trials with incorrect responses and with RTs longer than 3,500 ms or shorter than 500 ms (Cooper \& Shepard, 1975; de Lange, Hagoort, \& Toni, 2005; Heil \& Rolke, 2002; Kosslyn, DiGirolamo, Thompson, \& Alpert, 1998; Parsons, 1994; Sekiyama, 1982; Steggemann, Engbert, \& Weigelt, 2011; Wohlschläger \& Wohlschläger, 1998), with a total loss of $7.9 \%$ of the trials. RTs were analyzed by means of a fourway repeated measures analysis of variance (ANOVA) including Stroking (baseline, synchronous, asynchronous), stimulus Laterality (right, left), stimulus View (dorsum-view, palmview), and Rotation $\left(0^{\circ}\right.$, lateral, $180^{\circ}$, medial $)$ as the main factors. Lateral rotations included right hands (dorsum-view and palm-view) presented at $90^{\circ}$ and left hands (dorsum-view and palm-view) presented at $270^{\circ}$. Medial rotations included right hands (dorsum-view and palm-view) presented at $270^{\circ}$ and left hands (dorsum-view and palm-view) presented at $90^{\circ}$
(Coslett et al., 2010; Funk \& Brugger, 2008; ter Horst et al., 2010). Post-hoc comparisons were performed using the Newman-Keuls test $(p<.05)$.

Finally, we computed the correlation between the subjectively reported quality of the illusion (questionnaire) and behaviorally measured performance in the MR task. In particular, we correlated the distribution of the values of all the questionnaire items with the RTs of the MR task in the same condition (synchronous or asynchronous), separately for dorsum-view and palm-view hands. According to our hypothesis that the visuotactile stroking of the palmview should affect the MR of palm-view hands but not of dorsum-view hands, we ran the correlation analysis while keeping separated the RTs relative to MR of dorsum-view and palm-view hands. A Pearson correlation was performed between questionnaire values and MR RTs.

\section{Results}

Illusory ownership Agreement with the three target-Q items was stronger after the synchronous condition than after the asynchronous condition ( $p<.001$; see Fig. 3). Scores for the control-Q items did not show significant differences between synchronous and asynchronous stroking $(p>.07)$. In particular, subjects indicated stronger illusory touch $(\mathrm{Q} 1$, Q2) and self-attribution (Q3) during the synchronous stroking with respect to the asynchronous stroking (all $p \mathrm{~s}<.001$ ). No synchrony-related differences were found for any control-Q item (all ps > .16). Extending pilot data, these findings reveal that illusory ownership can be induced despite postural differences between the physical and fake hands.

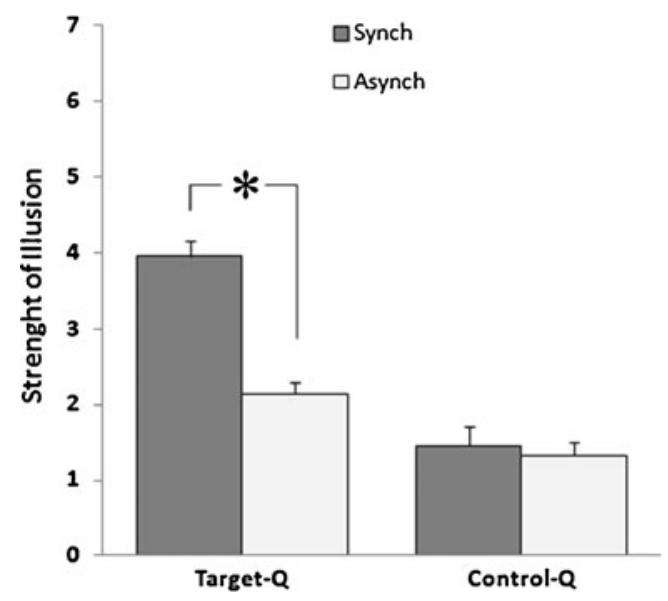

Fig. 3 Illusory ownership. Subjective reports showed that only responses to target-Q items (Questions 1-3, indicating self-attribution of the rubber hand) were stronger after the synchronous visuotactile stimulation. Responses to control-Q items (Questions 4 and 5) did not show any stimulation-related differences. The asterisk indicates significant difference, and error bars indicate standard errors 
Visuotactile stroking affects mental rotation Concerning the RTs in the MR task, we found a significant two-way interaction $[F(2,52)=9.7 ; p<.001]$ between stroking (synchronous, asynchronous) and view (palm-view, dorsum-view) caused by the significant RT difference between performance for the dorsum-view and palm-view that was only found in the synchronous condition (Fig. 4; dorsum-view, 1,078 ms; palm-view, 1,183 ms; $p<.001)$. In particular, only in the synchronous condition were RTs for hand stimuli shown in the palm-view slower with respect to dorsum-view stimuli. This difference was not significant in the baseline $(1,103$ and $1,115 \mathrm{~ms}$, respectively; $p=.4)$ and asynchronous $(1,084$ and $1,123 \mathrm{~ms}$, respectively; $p=.08)$ conditions. We interpret these data as a secondary effect of the establishment of the MOLA-like MR function (see the Discussion section).

The influence of visuotactile stroking on the MR of hands was further confirmed by the significant three-way interaction $[F(6,156)=2.3, p<.03]$ between stroking, view, and rotation (Fig. 5). Thus, the typical rotation-dependent distribution of RTs for the MR of hands (MOLA) was always present for dorsum-view stimuli (regardless of the stroking condition), but differed across palm-view conditions. For the dorsum-view stimuli, RTs increased for stimuli rotated from $0^{\circ}$ to $180^{\circ}$ and showed the longest RTs for stimuli at $180^{\circ}$, with respect to all of the other rotations (all $p \mathrm{~s}<.001$; Fig. 5). For the palm-view stimuli only following the synchronous condition, RTs for stimuli at $180^{\circ}$ and for lateral rotations were significantly longer with respect to $0^{\circ}$ and medial rotations (all $p \mathrm{~s}<.01$ ). Conversely, in the baseline and asynchronous conditions, the RTs for $180^{\circ}$ and lateral palm-view stimuli were not statistically different with respect to the other conditions (all $p \mathrm{~s}>.06$ ). These findings indicated that visuotactile stroking, which induced illusory hand ownership for a fake hand in palm-view, changed the

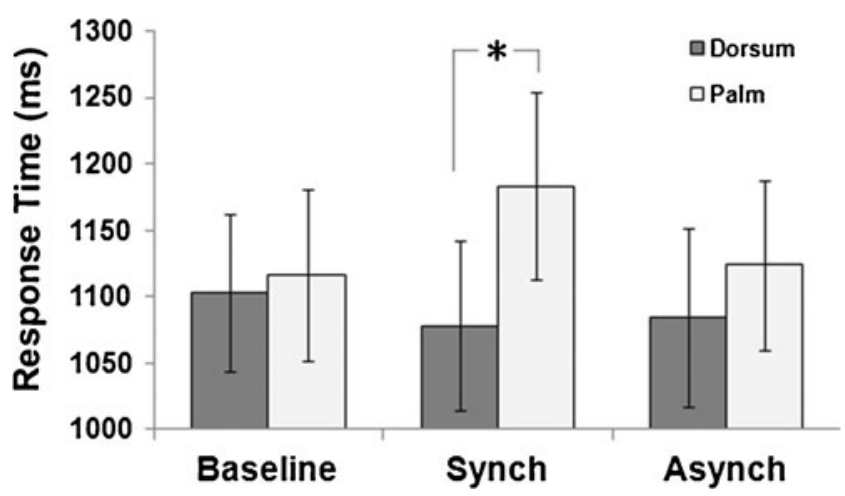

Fig. 4 View-dependent and synchrony-related effect of the rubber hand illusion on mental rotation (MR). The difference between the MR of the two stimulus views (dorsum-view or palm-view) was significant only during the synchronous visuotactile stimulation. During baseline and asynchronous stroking, there were no stimulus-viewrelated differences. The asterisk indicates significant difference, and error bars indicate standard errors
MR function for hand stimuli shown in palm-view but not for those in dorsum-view.

Furthermore, in all three experimental conditions the RTs for lateral rotations of dorsum-view stimuli were always faster, with respect to palm-view (baseline, 1,035 and 1,156 ms, respectively; synchronous, 1,008 and 1,290 ms, respectively; asynchronous, 1,041 and 1,207 ms, respectively; all $p \mathrm{~s}<.005)$. Finally, the RTs for medial rotations of dorsum-view and palm-view stimuli were not significantly different, regardless of stroking (all $p \mathrm{~s}>.8$ ).

Relation between RHI and MR We found that the subjectively reported sense of illusion correlated with performance in MR. In particular, there was a positive correlation $(r=$ $.37, p<.02)$ between hand ownership (Q3) after the synchronous condition and the MR speed for palm-view hands, and a negative correlation between hand ownership (Q3) and the MR speed for dorsum-view hands $(r=-.32$, $p<.04)$. In the asynchronous condition, hand ownership (Q3) did not correlate with MR of either palm-view nor dorsum-view hands (all $p s>.19)$.

Other effects The significant two-way interaction between view and rotation $[F(3,78)=15.8, p<.001]$ indicated that the difference in RTs for stimuli presented in the dorsumview and palm-view was significant for all of the rotations except medial ones. In detail, for stimuli at $0^{\circ}$ and lateral rotations, performance was faster with stimuli presented from the dorsum-view than with stimuli presented from the palm-view (for $0^{\circ}, 961$ and $1,074 \mathrm{~ms}$, respectively; for lateral, 1,082 and 1,218 ms, respectively; all $p \mathrm{~s}<.001)$. When the stimuli were presented upside down $\left(180^{\circ}\right)$, the direction of the difference was opposite: slower RTs for stimuli presented from the dorsum-view $(1,331 \mathrm{~ms})$, with respect to those in palm-view $(1,243 \mathrm{~ms} ; p<.01)$. For medial rotations, there was no significant difference between dorsum-view and palm-view. Finally, we found significant main effects of laterality $[F(1,26)=7.5, p<.01]$, view $[F(1,26)=4.6, p<.04]$, and rotation $[F(3,78)=39.5$, $p<.001]$. The main effect of laterality was accounted for by the faster performance for right $(1,092 \mathrm{~ms})$ than for left $(1,137 \mathrm{~ms})$ stimuli. The main effect of view was accounted for by faster responses for stimuli seen from the dorsum-view (1,088 ms) with respect to the palm-view $(1,140 \mathrm{~ms})$. The main effect of rotation was accounted for by the slowest performance for upside-down $\left(180^{\circ}\right)$ rotations $(1,288 \mathrm{~ms})$ and for lateral rotations $(1,123 \mathrm{~ms})$, as compared to medial rotations $(1,030 \mathrm{~ms})$ and the upright $\left(0^{\circ}\right)$ orientation $(1,013 \mathrm{~ms}$; all $p \mathrm{~s}<.001)$. The significant three-way interaction $[F(3,78)=6.8, p<.001]$ between laterality, view, and rotation indicated that for right stimuli presented at $0^{\circ}$ and lateral rotations, the dorsum-view produced faster performance than did the palm-view (all $p \mathrm{~s}<.01$ ). In addition, for $180^{\circ}$ 


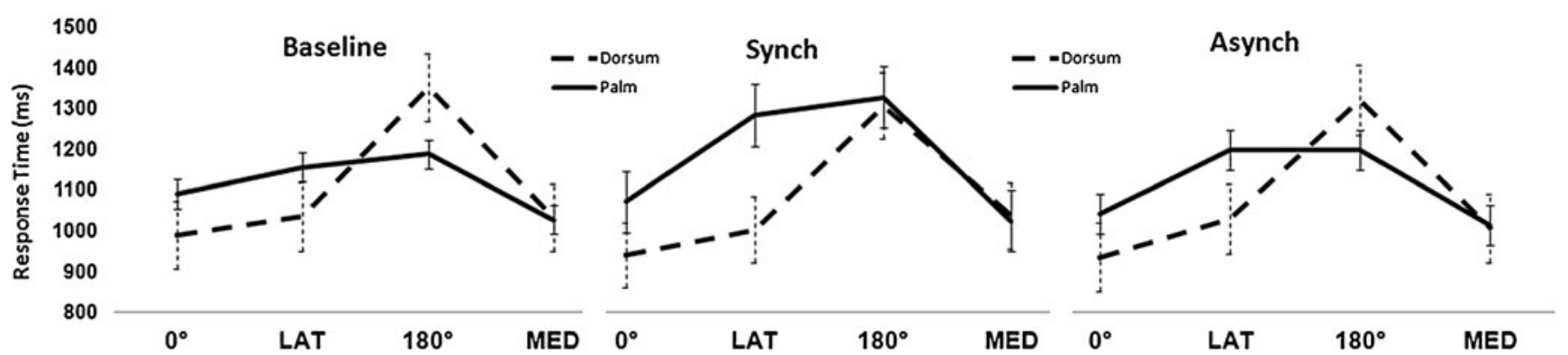

Fig. 5 Visuotactile stroking modality affected mental rotation's dependency on stimulus view and rotation. Mental rotation of palm-view hands was modulated by the visuotactile stimulation: only following synchronous stroking was subjects' performance

rotations, the dorsum-view was slower than the palm-view ( $p<$ $.001)$. Conversely, the stimulus view effect was much less pronounced for left-lateralized stimuli.

\section{Experiment 2}

Experiment 1 demonstrated that subjectively reported illusory ownership of a rubber hand can be manipulated by means of synchronous visuotactile stimulation, even when the subject's hand and the rubber hand are in incongruent postures. Some studies have indicated that subjective (e.g., questionnaire) and behavioral (e.g., illusory proprioceptive drift) measurements of the RHI have been associated with an experimentally induced illusory sense of ownership (Tsakiris \& Haggard, 2005). In order to further investigate the effect of our experimental setup — not only via subjective but also via a behavioral index of the RHI - we ran Experiment 2, which included a measurement of proprioceptive drift. It could be argued that both the proprioceptive drift and MR of hands are linked to proprioceptive mechanisms and recruit at least partially overlapping brain networks. Thus, it could be hypothesized that our experimental manipulation might affect not only the explicitly reported ownership of the rubber hand (questionnaires), but also the implicit illusory proprioceptive drift of perceived hand position. In particular, the felt hand position could be perceived as being closer to the rubber hand during the synchronous visuotactile stroking than during asynchronous stroking. We tested this hypothesis in Experiment 2, in which subjects were exposed to exactly the same experimental manipulations as in Experiment 1, but questionnaire as well as proprioceptive drift results were examined.

\section{Method}

A group of 12 subjects (4 female, 8 male) 20-24 years of age took part in Experiment 2. All of the subjects were right-handed more sensitive to rotations. There were no synchrony-related differences in the mental rotation of dorsum-view hands. Error bars indicate standard errors

(Briggs \& Nebes, 1975). The experimental setup and conditions replicated those of Experiment 1. Before the visuotactile stimulation the rubber hand was not visible, and a ruler was placed about $20 \mathrm{~cm}$ above the mirror to measure the initial subjectively perceived hand location (baseline). During the visuotactile stimulation, the ruler was removed and the rubber hand was visible (cf. the procedure of Exp. 1). After the visuotactile stimulation, the rubber hand was not visible anymore and the ruler was placed again above the mirror to measure the proprioceptive drift. Before and after the visuotactile stimulation, subjects were able to see the reflection of the ruler in the mirror and indicated the felt location of their right (stimulated) hand by verbally reporting the corresponding number on the ruler. The difference between the positions where the subjects localized their hands before and after the visuotactile stimulation indicated the condition-related proprioceptive drift (Lopez et al., 2010). The onsets of the ruler referred to different points and varied across conditions. Since the main question of Experiment 2 concerned any potential effect of visuotactile stimulation on the proprioceptive drift in our experimental setup, we did not measure MR.

Data analysis For the subjective experience of the RHI, the same analysis performed on the questionnaire data of Experiment 1 was carried out in Experiment 2. For the computation of proprioceptive drift, we calculated the amount of drift in a given condition by subtracting the position of the subject's hand before the stimulation from the position indicated by the subject after the stimulation. Subsequently, the proprioceptive drift data were analyzed by means of a $t$ test comparison between synchronous and asynchronous visuotactile stroking.

\section{Results}

Self-reported questionnaire data confirmed the findings of Experiment 1. Responses to the target-Q items indicated 
stronger illusions after synchronous visuotactile stroking, with respect to the asynchronous conditions. In particular, after the synchronous condition, subjects reported stronger illusory touch $(\mathrm{Q} 1, \mathrm{Q} 2)$ and self-attribution $(\mathrm{Q} 3)$ than after the asynchronous condition (all $p \mathrm{~s}<.04$ ). Responses to the control-Q items (Q5, Q5) did not vary as a function of visuotactile stimulation (all $p \mathrm{~s}>.1$ ). Confirming the data of Experiment 1, these findings showed that subjective experiences of the RHI can be induced even when the physical and fake hands are positioned in incongruent postures. Analysis of proprioceptive drift as an implicit index of the RHI indicated no significant difference between the synchronous $(-0.2 \mathrm{~cm})$ and asynchronous $(-1.2 \mathrm{~cm})$ stroking conditions $(p=.22)$. These findings are compatible with earlier observations that subjective and behavioral measurements related to the RHI can be dissociated, and they indicate that our experimental setup modulates subjectively-reported sense of illusion but not the proprioceptive drift.

\section{Discussion}

Visuotactile stimulation modulates mental rotation

The present investigation of the influence of visuotactile stimulation (manipulated according to the RHI paradigm) on MR of hands revealed three main findings. First, only after synchronous visuotactile stimulation was the MR of palm-view hands more dependent on the direction of rotation. Second, this synchrony-related effect was present for palmview but not for dorsum-view hands. Third, only after the synchronous condition was MR slower for the palm-view than for the dorsum-view hands.

We found that the rotation-dependent RT profile for the MR of palm-view stimuli was present only after synchronous visuotactile stimulation. Classically, the distribution of RTs for the MR of palm-view hands is weakly dependent on both stimulus orientation (Craje et al., 2010; Ionta \& Blanke, 2009) and the MOLA effect, relative to the direction of rotation (Coslett et al., 2010; ter Horst et al., 2010). This was confirmed in our baseline and asynchronous conditions - in which MR of palm-view hands was not found to depend on stimulus rotation - but the results differed after synchronous visuotactile stroking, becoming more dependent on the direction of MR. We argued that this synchrony-induced stronger dependency on rotation is reflected in the greater modulation of RTs for more awkward rotations $\left(180^{\circ}\right.$ and lateral) and is due to a stronger reliance on sensory-motor versus visual mechanisms during illusory hand ownership. Accordingly, the absence of a MOLA effect in the MR of palm-view hands has also been reported in patients suffering from sensorymotor pathologies, such as hemiparetic cerebral palsy (Craje et al., 2010), unilateral amelia (Funk \& Brugger, 2008), and chronic pain (Coslett et al., 2010), as well as in association with right-hemisphere lesions (Tomasino \& Rumiati, 2004). This suggests that the independence of the MR of palm-view hands from stimulus view/rotation - in the baseline and asynchronous conditions - might be due to the recruitment of visual rather sensory-motor mechanisms (Steenbergen, van Nimwegen, \& Craje, 2007; Wilson et al., 2004; Zacks \& Michelon, 2005). Interestingly, we found that only following synchronous visuotactile stimulation were RTs for palm-view stimuli presented at $180^{\circ}$ longer with respect to the baseline and asynchronous conditions. We suggest that the increase of RTs for this condition and stimulus could be due to a stronger reliance on sensory-motor rather than visual mechanisms, with a subsequent stronger dependence on biomechanical constraints, which in turn would result in longer RTs for more awkward rotations (as for $180^{\circ}$ ). This dissociation between sensory-motor and visual mechanisms in mental imagery has been demonstrated in several neurological conditions affecting the motor system and impairing motor but not visual imagery (Steenbergen et al., 2007; Tomasino \& Rumiati, 2004; Tomasino, Toraldo, \& Rumiati, 2003). If we consider these data together, one possible interpretation is that the illusory ownership of the fake hand (induced only by synchronous stroking) promoted the activation of sensory-motor rather than visually based mechanisms, leading to the observed stronger dependence on the direction of rotation. In addition, the synchrony-related establishment of a MOLAlike profile for MR was highly view-specific, being present only for the palm-view and not for the dorsum-view stimuli.

This result is further supported by our correlation analysis. As indicated by the correlation between subjectively reported hand ownership for the rubber hand and performance in the MR of palm-view hands, these findings suggest that the interaction between vision, touch, and proprioception manipulated during the RHI, leading to illusory hand ownership, is associated with changes in central body representations that further influence MR of hands. The notion that the RHI and MR of hands recruit similar mechanisms is supported by neuroimaging studies that have indicated stronger activity in partially overlapping regions, including mainly the premotor cortex and inferior parietal cortex. Premotor cortex has been consistently reported to be involved in MR (for a review, see Munzert et al., 2009) and in bodily illusions (Lenggenhager et al., 2011; Naito et al., 1999). Inferior parietal cortex has shown increasing activity as a function of spatial demands in MR (Wolbers, Weiller, \& Büchel, 2003), as well as during the induction phase of the RHI (Ehrsson et al., 2004, 2005). Additionally, if parietal cortex is targeted by transcranial magnetic stimulation, a consistent reduction of the RHI occurs (Kammers et al., 2009). However, to the best of our knowledge, no neuroimaging studies have yet directly compared brain activity relative to the RHI and the MR of body parts in a within-subjects 
design. Further developments of the present study will include the acquisition of such missing neuroimaging data.

From a behavioral point of view, the effects of the influence of vision and proprioception on MR of hands have been recently investigated in three separate groups of subjects performing MR of hands in three different experimental conditions (Shenton et al., 2004). The "fake hand" group kept their right hands out of sight either in a palm-up or palm-down posture, while a visible fake hand was positioned in either a palm-down or palm-up posture. The "proprioceptive" group kept their right hands out of sight in either a palm-down or palm-up posture, but there was no fake hand. The "control" group kept their right hands in view in either a palm-down or palm-up posture, and there was also no fake hand. In all three groups, RTs generally increased for palm-up stimuli when the subjects kept their hands palm down, but there were no condition-related differences (between groups). On the basis of these findings, Shenton et al. concluded that-regardless of the visual information from physical or fake hands-hand posture affects MR of hands that are shown in a congruent view. This result could be taken as evidence that the influence of proprioceptive information on $\mathrm{MR}$ of hands is relatively dominant over visual information.

Here, however, we showed that there is an interaction between proprioception and vision that depends on visuotactile stroking synchrony. We argue that two factors may explain the differences between these studies. First, the absence of visuotactile stroking may have crucially affected Shenton et al.'s (2004) results. Self-attribution of a fake hand is a fundamental aspect of the sense of ownership manipulated during the RHI by means of the synchrony between visual and tactile stroking. Illusion-related changes of the sense of ownership affect both behavior and brain activity (Tsakiris, 2010), with the patterns of activation being similar to the ones recruited by MR of body parts. Without visuotactile stroking (as in the setup of Shenton et al., 2004), the mere vision of a rubber hand is not sufficient to elicit the illusion and the consequential effects. This was also confirmed in our baseline and asynchronous conditions. The absence of an influence of vision on MR of hands that was reported by Shenton et al. could thus depend on this lack of illusory hand ownership. Second, employing a between-subjects design hampered direct within-subjects comparisons of the different experimental conditions and may have invalidated potentially small effects of the postural manipulations on MR in the study of Shenton et al. We reduced the effects of intersubject variability on the analyses and increased the statistical power of our comparisons by using a within-subjects design and asking all of our subjects to perform MR of hands after each experimental condition. In addition, we adopted the standard RHI protocol, including a manipulation of visuotactile stroking in terms of either a synchronous or asynchronous relationship between the visual stroking (on the rubber hand) and tactile stroking (on the subject's hand). Finally, we compared both experimental conditions with a baseline condition in which no stroking was applied to the subject's hand. These manipulations allowed for a more reliable and direct comparison of the effects of the experimental conditions and revealed a robust visuotactile effect on MR of hands.

It might be argued that the increase of RTs for the palmview hands following the synchronous visuotactile stroking suggests that illusory ownership of the fake hand in a palmup position does not facilitate MR of hand stimuli presented in the congruent view, but rather interferes with the task. However, if we also take into account the MOLA-like distribution of RTs, again only following the synchronous condition, we can interpret these data as a secondary effect of the establishment of the (usually absent) MR sensorymotor functions for the palm-view stimuli. Accordingly, we argue that the general synchrony-related effect of slowing down in the MR of palm-view hands is due to and reflects the increase in RTs for stimuli presented in more awkward rotations.

It could be also argued that during the synchronous condition, two kinds of proprioceptive hand representations are activated - the physical hand's position (palm-down posture) and the illusory hand position (palm-up) — and that this coactivation of two hand representations leads to similar MR profiles for the dorsum-view and palm-view hands. The activation of sensory-motor mechanisms during MR can be deduced from longer RTs for more awkward MRs (MOLA effect), while a weaker MOLA effect can be associated with the activation of more visually-based mechanisms. Thus, we argue that in the baseline and asynchronous conditions, the palm-view hands were mentally rotated with a weaker reliance on sensory-motor mechanisms, while such reliance was stronger for mentally rotating the same stimuli in the synchronous condition. For the dorsum-view hands the MOLA effect is present in all conditions, and it is thus likely that sensorymotor mechanisms are always activated for this kind of stimuli. However the possibility of a coexistence of two proprioceptive representations could result in proprioceptive conflict, leading to condition-related behavioral differences also for the dorsum-view stimuli. Future studies will be required to investigate this possibility further.

Illusory ownership for a fake hand in an incongruent posture

Our data are also of relevance to previous work on illusory hand ownership. The present findings show that even if the fake hand is positioned palm-up and the subject's hand is palm-down (postural incongruence), selfattribution measured by the standard questionnaire is stronger during synchronous visuotactile stimulation, with respect 
to asynchronous stimulation. We propose that illusory hand ownership is made possible, despite this postural incongruence (which normally reduces or abolishes the RHI), by what we term the "anatomical plausibility" of the rubber hand's posture with respect to the physical arm (Armel \& Ramachandran, 2003; Farnè, Pavani, Meneghello, \& Làdavas, 2000; Pavani et al., 2000). We define "anatomical plausibility" as the anatomical ease of matching the posture of the fake hand (not only the general possibility to assume a specific posture). In our setup, the fake right hand was in a palm-up posture and was observed as being touched on the palm-view (visual stroking), while the subjects were also stimulated on their right palm, but in a palm-down posture (tactile stroking). Thus, despite the postural incongruence between the real and fake hands, the subjectively reported experience of illusory hand ownership was stronger during synchronous than during asynchronous visuotactile stimulation. This result seems in conflict with previous findings on the effect of positional congruence in the RHI. Thus, some studies have reported that if the fake hand is positioned in an incongruent alignment with respect to the subject's hand, illusory self-attribution decreases (Ehrsson et al., 2004; Lloyd, 2007). However, it has been shown that in order to induce stroking-related self-attribution of a fake hand, it is sufficient to place the fake hand within the peripersonal space (for a review, see Makin et al., 2008). Moreover, people can experience the RHI even if the fake hand (or someone else's hand) is displayed in a video (Schaefer, Noennig, Heinze, \& Rotte, 2006) or in a virtual-reality setting (Slater, Perez-Marcos, Ehrsson, \& Sanchez-Vives, 2008). This supports the notion that the anatomical plausibility of the fake hand's posture, more than congruence with the subject's hand position per se, could be a decisive factor in eliciting the RHI.

With respect to our results, it is worth noting that, while in previous studies the dependency of the fake hand's selfattribution on its alignment with the subject's hand was investigated only for localizations of the fake hand in the horizontal plane (Costantini \& Haggard, 2007; Lloyd, Morrison, \& Roberts, 2006; Tsakiris \& Haggard, 2005), in our experiment the manipulation was on the $z$-axis (palm-up and palm-down). According to this account, one possibility is that the onset of the RHI in our setup could be due to an additional illusory postural recalibration that would interact with the visual recalibration of the hand position classically investigated in previous studies. Our data show that in our RHI setup there is an illusory change in the perceived posture of the hand. We propose that two different domains of congruence are manipulated in the present versus in previous setups. When the fake hand is moved away with respect to the subject's body (as with changes in the horizontal plane), visual congruency is affected. When the position of the rubber hand remains the same but its posture changes, postural congruency is affected instead. Thus, during the RHI the anatomical plausibility of the fake hand, in addition to its position, is crucial for the illusion to take place. The difference between visual and postural congruence can explain how and why under some circumstances the RHI can be induced, even if it is in a visually incongruent but anatomically plausible (posture) congruent position with respect to the subject's hand. In findings compatible with the present study, but in the absence of systematically manipulated visual input, previous authors have shown illusory changes in perceived posture following tendon vibration of the wrist (Naito, Roland, \& Ehrsson, 2002) as well as of the biceps muscles (Lackner, 1988). We propose that when visual and proprioceptive inputs are both available - as in the RHI setup - the integration of vision and proprioception leading to the illusion depends on anatomical plausibility, further driven by visuotactile synchrony. In other words, if the rubber hand lies in a visually and posturally possible position, and if visual and tactile stimulations are synchronous, then the RHI is likely to take place, leading to an illusory body posture. However, more experiments will be required to disentangle the effects due to illusory postural changes from the effects due merely to the RHI.

\section{Subjective versus behavioral measurements of the RHI}

In Experiment 2, we found that following synchronous visuotactile stimulation, subjects' responses indicated stronger selfattribution of the rubber hand, as compared to the asynchronous condition. This was not the case for proprioceptive drift. We note, however, that proprioceptive drift has been reported even in the absence of illusory ownership (Holmes, Snijders, \& Spence, 2006), that both measures can be experimentally dissociated (Holle, McLatchie, Maurer, \& Ward, 2011; Rohde, Di Luca, \& Ernst, 2011), and that some authors only acquired subjective data (questionnaires; e.g., Ehrsson et al., 2004; Holmes et al., 2006; Lloyd, 2007), whereas others only reported behavioral data (proprioceptive drift; e.g., Costantini \& Haggard, 2007; Tsakiris, Costantini, \& Haggard, 2008). The present data are thus compatible with previous accounts of a weak link between proprioceptive drift and selfattribution (i.e., Makin et al., 2008), suggesting that these measures concern related but distinct mechanisms. The changes in body ownership induced by the RHI have been quantified by a wide range of implicit measurements, including skin conductance (Armel \& Ramachandran, 2003), cooling of skin temperature (Moseley et al., 2008), visuotactile perception (Aspell, Lenggenhager, \& Blanke, 2009; Zopf, Savage, \& Williams, 2010), changes in heartbeat frequency (Tsakiris, Tajadura-Jimenez, \& Costantini, 2011), and histamine reactivity (Barnsley et al., 2011). Based on our results showing changes in MR performance under conditions that also induced changes in body ownership, we propose that (1) MR and the RHI share sensory-motor and multisensory mechanisms and (2) the speed of MR - and in particular, the 
magnitude of the interaction between the direction of MR and the stimulus view-should be added to the list of behavioral measures of the RHI. Such ownership-dependent changes in MR speed would have the advantage over earlier classical RHI measures such as proprioceptive drift, in that MR is based on repeated RT and accuracy measures.

Author note The authors thank Mario Prsa for his invaluable suggestions. O.B. is supported by the European Science Foundation, the Swiss National Science Foundation, and the Bertarelli Foundation.

\section{References}

Aimola Davies, A. M., White, R. C., Thew, G., Aimola, N. M. V., \& Davies, M. (2010). Visual capture of action, experience of ownership, and the illusion of self-touch: A new rubber hand paradigm. Perception, 39, 830-838. doi:10.1068/p6610

Armel, K. C., \& Ramachandran, V. S. (2003). Projecting sensations to external objects: Evidence from skin conductance response. Proceedings of the Royal Society B, 270, 1499-1506.

Aspell, J. E., Lenggenhager, B., \& Blanke, O. (2009). Keeping in touch with one's self: Multisensory mechanisms of self-consciousness. PLoS One, 4, e6488. doi:10.1371/journal.pone.0006488

Barnsley, N., McAuley, J. H., Mohan, R., Dey, A., Thomas, P., \& Moseley, G. L. (2011). The rubber hand illusion increases histamine reactivity in the real arm. Current Biology, 21, 945946.

Berlucchi, G., \& Aglioti, S. (1997). The body in the brain: Neural bases of corporeal awareness. Trends in Neurosciences, 20, 560564.

Blanke, O., \& Metzinger, T. (2009). Full-body illusions and minimal phenomenal selfhood. Trends in Cognitive Sciences, 13, 7-13.

Bonda, E., Petrides, M., Frey, S., \& Evans, A. (1995). Neural correlates of mental transformations of the body-in-space. Proceedings of the National Academy of Sciences, 92, 11180-11184.

Botvinick, M., \& Cohen, J. (1998). Rubber hands "feel" touch that eyes see. Nature, 391, 756.

Briggs, G. G., \& Nebes, R. D. (1975). Patterns of hand preference in a student population. Cortex, 11, 230-238.

Cooper, L. A., \& Shepard, R. N. (1975). Mental transformations in the identification of left and right hands. Journal of Experimental Psychology. Human Perception and Performance, 1, 48-56. doi:10.1037/0096-1523.1.1.48

Corballis, M. C., \& Sergent, J. (1989). Hemispheric specialization for mental rotation. Cortex, 25, 15-25.

Corradi-Dell'Acqua, C., \& Tessari, A. (2010). Is the body in the eye of the beholder? Visual processing of bodies in individuals with anomalous anatomical sensory and motor features. Neuropsychologia, 48, 689-702.

Coslett, H. B., Medina, J., Kliot, D., \& Burkey, A. R. (2010). Mental motor imagery indexes pain: The hand laterality task. European Journal of Pain, 14, 1007-1013.

Costantini, M., \& Haggard, P. (2007). The rubber hand illusion: Sensitivity and reference frame for body ownership. Consciousness and Cognition, 16, 229-240.

Craje, C., van Elk, M., Beeren, M., van Schie, H. T., Bekkering, H., \& Steenbergen, B. (2010). Compromised motor planning and Motor Imagery in right Hemiparetic Cerebral Palsy. Research into Developmental Disabilities, 31, 1313-1322.

Damasio, A. R. (2000). The feeling of what happens: Body and emotion in the making of consciousness. New York, NY: Harcourt Brace. de Lange, F. P., Hagoort, P., \& Toni, I. (2005). Neural topography and content of movement representations. Journal of Cognitive Neuroscience, 17, 97-112.

de Lange, F. P., Helmich, R. C., \& Toni, I. (2006). Posture influences motor imagery: An fMRI study. NeuroImage, 33, 609-617.

Decety, J., Jeannerod, M., \& Prablanc, C. (1989). The timing of mentally represented actions. Behavioural Brain Research, 34, 35-42. doi:10.1016/S0166-4328(89)80088-9

Ehrsson, H. H., Holmes, N. P., \& Passingham, R. E. (2005). Touching a rubber hand: Feeling of body ownership is associated with activity in multisensory brain areas. Journal of Neuroscience, $25,10564-10573$.

Ehrsson, H. H., Spence, C., \& Passingham, R. E. (2004). That's my hand! Activity in premotor cortex reflects feeling of ownership of a limb. Science, 305, 875-877.

Farnè, A., Pavani, F., Meneghello, F., \& Làdavas, E. (2000). Left tactile extinction following visual stimulation of a rubber hand. Brain, 123, 2350-2360.

Fourkas, A. D., Ionta, S., \& Aglioti, S. M. (2006). Influence of imagined posture and imagery modality on corticospinal excitability. Behavioural Brain Research, 168, 190-196.

Funk, M., \& Brugger, P. (2008). Mental rotation of congenitally absent hands. Journal of the International Neuropsychological Society, 14, 81-89.

Gallagher, S. (2005). How the body shapes the mind. Oxford, U.K.: Oxford University Press, Clarendon Press.

Gawryszewski, L. G., Silva-dos-Santos, C. F., Santos-Silva, J. C., Lameira, A. P., \& Pereira, A., Jr. (2007). Mental rotation of anthropoid hands: A chronometric study. Brazilian Journal of Medical and Biological Research, 40, 377-381.

Gerardin, E., Sirigu, A., Lehéricy, S., Poline, J. B., Gaymard, B., Marsault, C., . . . Le Bihan, D. (2000). Partially overlapping neural networks for real and imagined hand movements. Cerebral Cortex, 10, 1093-1104.

Grèzes, J., \& Decety, J. (2001). Functional anatomy of execution, mental simulation, observation, and verb generation of actions: A meta-analysis. Human Brain Mapping, 12, 1-19.

Haggard, P., Taylor-Clarke, M., \& Kennett, S. (2003). Tactile perception, cortical representation and the bodily self. Current Biology, 13, R170-R173.

Heil, M., \& Rolke, B. (2002). Toward a chronopsychophysiology of mental rotation. Psychophysiology, 39, 414-422.

Holle, H., McLatchie, N., Maurer, S., \& Ward, J. (2011). Proprioceptive drift without illusions of ownership for rotated hands in the "rubber hand illusion" paradigm. Cognitive Neuroscience, 2, 171178.

Holmes, N. P., Snijders, H. J., \& Spence, C. (2006). Reaching with alien limbs: Visual exposure to prosthetic hands in a mirror biases proprioception without accompanying illusions of ownership. Perception \& Psychophysics, 68, 685-701. doi:10.3758/ BF03208768

Ionta, S., \& Blanke, O. (2009). Differential influence of hands posture on mental rotation of hands and feet in left and right handers. Experimental Brain Research, 195, 207-217.

Ionta, S., Ferretti, A., Merla, A., Tartaro, A., \& Romani, G. L. (2010). Step-by-step: The effects of physical practice on the neural correlates of locomotion imagery revealed by fMRI. Human Brain Mapping, 31, 694-702.

Ionta, S., Fourkas, A. D., Fiorio, M., \& Aglioti, S. M. (2007). The influence of hands posture on mental rotation of hands and feet. Experimental Brain Research, 183, 1-7. doi:10.1007/s00221-007-1020-2

Ionta, S., Perruchoud, D., Draganski, B., \& Blanke, O. (2012). Body context and posture affect mental imagery of hands. PLoS One, 7, e34382.

Jeannerod, M. (2006). Motor cognition: What actions tell the self. Oxford, U.K.: Oxford University Press. 
Jeannerod, M. (2007). Being oneself. The Journal of Physiology, 101, $161-168$.

Kammers, M. P., Verhagen, L., Dijkerman, H. C., Hogendoorn, H., De Vignemont, F., \& Schutter, D. J. (2009). Is this hand for real? Attenuation of the rubber hand illusion by transcranial magnetic stimulation over the inferior parietal lobule. Journal of Cognitive Neuroscience, 21, 1311-1320.

Kosslyn, S. M., DiGirolamo, G. J., Thompson, W. L., \& Alpert, N. M. (1998). Mental rotation of objects versus hands: Neural mechanisms revealed by positron emission tomography. Psychophysiology, 35, 151-161.

Lackner, J. R. (1988). Some proprioceptive influences on the perceptual representation of body shape and orientation. Brain, 111, 281-297.

Lenggenhager, B., Halje, P., \& Blanke, O. (2011). Alpha band oscillations correlate with illusory self-location induced by virtual reality. European Journal of Neuroscience, 33, 1935-1943.

Lloyd, D. M. (2007). Spatial limits on referred touch to an alien limb may reflect boundaries of visuo-tactile peripersonal space surrounding the hand. Brain and Cognition, 64, 104-109. doi:10.1016/j.bandc.2006.09.013

Lloyd, D., Morrison, I., \& Roberts, N. (2006). Role for human posterior parietal cortex in visual processing of aversive objects in peripersonal space. Journal of Neurophysiology, 95, 205-214. doi:10.1152/jn.00614.2005

Lopez, C., Lenggenhager, B., \& Blanke, O. (2010). How vestibular stimulation interacts with illusory hand ownership. Conscious and Cognition, 19, 33-47.

Makin, T. R., Holmes, N. P., \& Ehrsson, H. H. (2008). On the other hand: Dummy hands and peripersonal space. Behavioural Brain Research, 191, 1-10.

Makin, T. R., Holmes, N. P., \& Zohary, E. (2007). Is that near my hand? Multisensory representation of peripersonal space in human intraparietal sulcus. Journal of Neuroscience, 27, 731-740.

Moseley, G. L., Olthof, N., Venema, A., Don, S., Wijers, M., Gallace, A., \& Spence, C. (2008). Psychologically induced cooling of a specific body part caused by the illusory ownership of an artificial counterpart. Proceedings of the National Academy of Sciences, 105, 13169-13173. doi:10.1073/pnas.0803768105

Munzert, J., Lorey, B., \& Zentgraf, K. (2009). Cognitive motor processes: The role of motor imagery in the study of motor representations. Brain Research Reviews, 60, 306-326.

Munzert, J., \& Zentgraf, K. (2009). Motor imagery and its implications for understanding the motor system. Progress in Brain Research, 174. 219-229. doi:10.1016/S0079-6123(09)01318-1

Naito, E., Ehrsson, H. H., Geyer, S., Zilles, K., \& Roland, P. E. (1999). Illusory arm movements activate cortical motor areas: A positron emission tomography study. Journal of Neuroscience, 19, 6134 6144.

Naito, E., Roland, P. E., \& Ehrsson, H. H. (2002). I feel my hand moving: A new role of the primary motor cortex in somatic perception of limb movement. Neuron, 36, 979-988.

Overney, L. S., \& Blanke, O. (2009). Impaired imagery for upper limbs. Brain Topography, 22, 27-43.

Palluel, E., Aspell, J. E., \& Blanke, O. (2011). Leg muscle vibration modulates bodily self-consciousness: Integration of proprioceptive, visual, and tactile signals. Journal of Neurophysiology, 105, 2239-2247.

Parsons, L. M. (1987a). Imagined spatial transformation of one's body. Journal of Experimental Psychology. General, 116, 172-191. doi:10.1037/0096-3445.116.2.172

Parsons, L. M. (1987b). Imagined spatial transformations of one's hands and feet. Cognitive Psychology, 19, 178-241.

Parsons, L. M. (1994). Temporal and kinematic properties of motor behavior reflected in mentally simulated action. Journal of
Experimental Psychology. Human Perception and Performance, 20, 709-730. doi:10.1037/0096-1523.20.4.709

Parsons, L. M., Fox, P. T., Downs, J. H., Glass, T., Hirsch, T. B., Martin, C. C., . . Lancaster, J. L. (1995). Use of implicit motor imagery for visual shape discrimination as revealed by PET. Nature, 375, 54-58.

Pavani, F., Spence, C., \& Driver, J. (2000). Visual capture of touch: Out-of-the-body experiences with rubber gloves. Psychological Science, 11, 353-359.

Petit, L. S., Pegna, A. J., Mayer, E., \& Hauert, C. A. (2003). Representation of anatomical constraints in motor imagery: Mental rotation of a body segment. Brain and Cognition, 51, 95-101.

Porro, C. A., Francescato, M. P., Cettolo, V., Diamond, M. E., Baraldi, P., Zuiani, C., . . . di Prampero, P. E. (1996). Primary motor and sensory cortex activation during motor performance and motor imagery: A functional magnetic resonance imaging study. Journal of Neuroscience, 16, 7688-7698.

Rohde, M., Di Luca, M., \& Ernst, M. O. (2011). The rubber hand illusion: Feeling of ownership and proprioceptive drift do not go hand in hand. PLoS One, 6, e21659.

Schaefer, M., Noennig, N., Heinze, H.-J., \& Rotte, M. (2006). Fooling your feelings: Artificially induced referred sensations are linked to a modulation of the primary somatosensory cortex. NeuroImage, 29, $67-73$.

Sekiyama, K. (1982). Kinesthetic aspects of mental representations in the identification of left and right hands. Perception \& Psychophysics, 32, 89-95.

Sforza, A., Bufalari, I., Haggard, P., \& Aglioti, S. M. (2010). My face in yours: Visuo-tactile facial stimulation influences sense of identity. Social Neuroscience, 5, 148-162.

Shenton, J. T., Schwoebel, J., \& Coslett, H. B. (2004). Mental motor imagery and the body schema: Evidence for proprioceptive dominance. Neuroscience Letters, 370, 19-24.

Shepard, R. N., \& Metzler, J. (1971). Mental rotation of three-dimensional objects. Science, 171, 701-703. doi:10.1126/science.171.3972.701

Sirigu, A., \& Duhamel, J. R. (2001). Motor and visual imagery as two complementary but neurally dissociable mental processes. Journal of Cognitive Neuroscience, 13, 910-919.

Sirigu, A., Duhamel, J. R., Cohen, L., Pillon, B., Dubois, B., \& Agid, Y. (1996). The mental representation of hand movements after parietal cortex damage. Science, 273, 1564-1568.

Slater, M., Perez-Marcos, D., Ehrsson, H. H., \& Sanchez-Vives, M. V. (2008). Towards a digital body: The virtual arm illusion. Frontiers in Human Neuroscience, 2, 6.

Steenbergen, B., van Nimwegen, M., \& Craje, C. (2007). Solving a mental rotation task in congenital hemiparesis: Motor imagery versus visual imagery. Neuropsychologia, 45, 3324-3328.

Steggemann, Y., Engbert, K., \& Weigelt, M. (2011). Selective effects of motor expertise in mental body rotation tasks: Comparing object-based and perspective transformations. Brain and Cognition, 76, 97-105.

ter Horst, A. C., Jongsma, M. L., Janssen, L. K., van Lier, R., \& Steenbergen, B. (2012). Different mental rotation strategies reflected in the rotation related negativity. Psychophysiology, 49, $566-573$.

ter Horst, A. C., van Lier, R., \& Steenbergen, B. (2010). Mental rotation task of hands: Differential influence number of rotational axes. Experimental Brain Research, 203, 347-354.

Thayer, Z. C., Johnson, B. W., Corballis, M. C., \& Hamm, J. P. (2001). Perceptual and motor mechanisms for mental rotation of human hands. NeuroReport, 12, 3433-3437.

Tomasino, B., \& Rumiati, R. I. (2004). Effects of strategies on mental rotation and hemispheric lateralization: Neuropsychological evidence. Journal of Cognitive Neuroscience, 16, 878-888.

Tomasino, B., Toraldo, A., \& Rumiati, R. I. (2003). Dissociation between the mental rotation of visual images and motor images 
in unilateral brain-damaged patients. Brain and Cognition, 51, 368-371.

Tsakiris, M. (2010). My body in the brain: A neurocognitive model of body-ownership. Neuropsychologia, 48, 703-712. doi:10.1016/ j.neuropsychologia.2009.09.034

Tsakiris, M., Costantini, M., \& Haggard, P. (2008). The role of the right temporo-parietal junction in maintaining a coherent sense of one's body. Neuropsychologia, 46, 3014-3018. doi:10.1016/ j.neuropsychologia.2008.06.004

Tsakiris, M., \& Haggard, P. (2005). The rubber hand illusion revisited: Visuotactile integration and self-attribution. Journal of Experimental Psychology. Human Perception and Performance, 31, 80-91.

Tsakiris, M., Tajadura-Jimenez, A., \& Costantini, M. (2011). Just a heartbeat away from one's body: Interoceptive sensitivity predicts malleability of body-representations. Proceedings of the Royal Society B, 278, 2470-2476.
Wilson, P. H., Maruff, P., Butson, M., Williams, J., Lum, J., \& Thomas, P. R. (2004). Internal representation of movement in children with developmental coordination disorder: A mental rotation task. Developmental Medicine and Child Neurology, 46, 754-759.

Wohlschläger, A., \& Wohlschläger, A. (1998). Mental and manual rotation. Journal of Experimental Psychology. Human Perception and Performance, 24, 397-412. doi:10.1037/0096-1523.24.2.397

Wolbers, T., Weiller, C., \& Büchel, C. (2003). Contralateral coding of imagined body parts in the superior parietal lobe. Cerebral Cortex, 13, 392-399.

Zacks, J. M., \& Michelon, P. (2005). Transformations of visuospatial images. Behavioral and Cognitive Neuroscience Reviews, 4, 96118.

Zopf, R., Savage, G., \& Williams, M. A. (2010). Crossmodal congruency measures of lateral distance effects on the rubber hand illusion. Neuropsychologia, 48, 713-725. 